\title{
A Nanogel with Effective Blood-Brain Barrier Penetration Ability through Passive and Active Dual-Targeting Function
}

\author{
Jun Liu, ${ }^{1}$ Meng Li, ${ }^{1}$ Yong Huang, ${ }^{2}$ Li Zhang, ${ }^{2}$ Wei Li, ${ }^{2}$ Peng Cao $\mathbb{D}^{1},{ }^{1}$ Weijie Min $\mathbb{D},{ }^{3}$ \\ Jingyuan $\mathrm{Li}^{1}{ }^{1}$ and Wei Jing ${ }^{4}{ }^{4}$ \\ ${ }^{1}$ Department of Neurosurgery, General Hospital of Northern Theater Command, Postgraduate Training Base of General Hospital of \\ Northern Theater Command of Jinzhou Medical University, No. 83, Wenhua Road, Shenhe District, Shenyang, China \\ ${ }^{2}$ Department of Nanomedicine \& Shanghai Key Laboratory of Cell Engineering, Naval Medical University, Shanghai 200433, China \\ ${ }^{3}$ Department of Neurosurgery, Changhai Hospital, Naval Medical University, Shanghai 200433, China \\ ${ }^{4}$ Department of Hepatobiliary Pancreatic Surgery, Changhai Hospital, Naval Medical University, Shanghai 200433, China
}

Correspondence should be addressed to Peng Cao; 16031629@qq.com, Weijie Min; min_203@126.com, and Wei Jing; jingwei7777@163.com

Received 30 December 2020; Accepted 30 October 2021; Published 24 December 2021

Academic Editor: Hassan Karimi-Maleh

Copyright ( 92021 Jun Liu et al. This is an open access article distributed under the Creative Commons Attribution License, which permits unrestricted use, distribution, and reproduction in any medium, provided the original work is properly cited.

\begin{abstract}
Clinically, surgery assisted by chemotherapy is the most effective treatment of cancer. But from our clinical observation, the median survival of patients with glioblastoma is still not so good with only 15-16 months. The low therapeutic index is mainly due to the blood-brain barrier (BBB) which significantly hindered the chemotherapeutic drug accumulation in tumor tissue. One main composition of the BBB is astrocyte, which contains a lipophilic cell membrane, which prevents more than $98 \%$ of small-molecule drugs from entering the brain. Previously, we found that the nanogel with passive targeting function can increase the $\mathrm{BBB}$ penetration ability, which indicates that it could be used to overcome the above mentioned in vivo obstacles which promoted drug accumulation in the tumor. In this study, thermosensitive targeted nanogel delivery systems (DPPC) with cell-penetrating peptides (CPP) are introduced onto the particle surface for active astrocyte breaking. The hydrodynamic radius of DPPC is around $300 \mathrm{~nm}$, the potential is about $0-5 \mathrm{mV}$, and the TEM and DLS studies further confirm its well spherical morphology and uniform distribution. The DPPC is verified as the biocompatible carriers for further application by cell viability tests. The in vitro-constructed BBB model successfully proves that DPPC can efficiently penetrate the BBB, which is attributed to both the temperature-sensitive passive targeting and the active CPP penetration. Consequently, the intracellular doxorubicin (DOX) promotes such functional DPPC at the relatively high temperature inside tumor microenvironment (TME) $\left(\sim 42^{\circ} \mathrm{C}\right)$, which obviously improves intratumor drug accumulation and tumor cell-killing effects. The dual-targeted nanogel delivery systems designed in this study provides a more effective strategy for the treatment of glioma.
\end{abstract}

\section{Introduction}

Gliomas account for about 80 percent of all tumors in the brain with an occurrence of 7 of 100,000 worldwide. Most of the gliomas are so-called diffuse, which are characterized by diffuse infiltration of tumor cells in the brain tissue $[1,2]$. According to the cell phenotype, diffuse gliomas are classified as different types in the WHO 2007 which includes astrocytes, oligodendrocytes, or oligodendrocyte tumors [3-5]. Although the malignant brain cancer can be suppressed by current clinical treatments for like a combination of surgery, radiotherapy, and chemotherapy, it is difficult to completely be ablated by surgery due to above mentioned diffuse infiltration. Chemotherapy is thus an effective treatment for promoting malignant brain cancer $[1,6,7]$. However, the function of the central nervous system (CNS) blood vessels is essential for full brain function, not only to effectively provide nutrients and oxygen to the brain but also to protect the brain from potential neurotoxic compounds, a protective blood barrier known as the blood-brain barrier (BBB) [8-11]. The therapeutic efficiency of chemotherapeutic drugs is thus limited due to this BBB. The BBB consists mainly of tightly sealed cerebral capillary 
endothelial cells (BCEC), which are surrounded by the end of the astrocytic perivascular vessels and the pericytes [12-17].

On the other hand, both the lipid nature of BCEC and the transport systems in the lumen as well as the lumen membrane provide an opportunity to transport substrates to the brain. In addition, the high metabolic demand of high-grade glioma generates the hypoxic region, which results in the increase of vascular endothelial growth factor (VEGF) level and angiogenesis [18, 19]. All these factors contributed to the formation of abnormal blood vessels and the dysfunction of the blood-brain-tumor barrier (BBTB). However, even if the BBTB is considered "leaky" in the core of high-grade glioblastoma, the scale of this local damage is unlikely to be large enough to allow a meaningful amount of drug penetration. In addition, for most glioblastomas, especially for low-grade diffuse gliomas, the BBTB behaves like the complete blood-brain barrier (BBB) and blocks the effective passage of cancer drugs, including small molecules and antibodies [13, 20-23]. Therefore, the BBB and BBTB are major barriers which hinder the treatment of brain tumors both in low- and high-grade gliomas.

Consequently, it is an urgent problem to overcome the blood-brain barrier and consider the side effects of the drug delivery system and the effective drug delivery volume. Many strategies have been developed for overcoming the BBB and/ or BBTB referring to their structural characteristics. The methods mainly include the penetration destruction of $\mathrm{BBB}$, interleukin receptor-mediated BBTB opening, inhibition of drug efflux transporters, and receptor-mediated transport delivery systems [24-28]. Among which, the nanosized delivery systems including polymer nanogels, micelles, liposomes, protein nanocages, and inorganic nanoparticles have been extensively reported for effective systemic brain tumor delivery using well-established endogenous $\mathrm{BBB}$ transport pathways [29-40]. Our team has a lot of experience in the field of nanosized delivery system research $[41,42]$. In our previous study, we found that the thermosensitive poly $(\mathrm{N}$-isopropylacrylamide)-based nanogel with the volume phase change temperature (VPTT) around $38^{\circ} \mathrm{C}$ was an excellent candidate for brain tumor drug delivery system. This system successfully overcame the $\mathrm{BBB}$ and transported the protein drug with high therapeutic efficiency due to its high in vivo/vitro stable structure and its thermosensitive passive targeting function (TSPT) [42-44].

For further promoting the drug delivery efficiency, it is highly desired to develop a nanogel with intelligent active targeting properties or by the fine carrier's tailoring referring to the classic "Li-Teruo" plot $[31,32,42,45]$, which can be utilized to increase the BBB and or BBTB crossing ability. It has been reported that cell penetration peptide (CPP, a short amphiphilic or cationic peptide) has a high BBB crossing ability [45-47]. Thus, this CPP can be utilized to conjugate with the PNIPAM-based nanogel to obtain a nanogel system with passive and active dual targeting function.

Based on the physiological structure of the blood-brain barrier (BBB) and our previous studies, in this paper, a passive and active dual targeting nanogel will be prepared by conjugating the thermosensitive polymer PNIPAM to the side chain of cationic polymer PEI and linking the CPP on the surface. Then, the chemotherapeutic drug doxorubicin (DOX) will be loaded into the nanogel resulting in an intelligent delivery system DOX@PNIPAM-PEI-CPP (DPPC). More importantly, the $\mathrm{BBB}$ penetrability mechanism of DPPC will be detailedly investigated and illustrated by using an in vitro $\mathrm{BBB}$ model. The drug delivery system designed in this study provides a new therapeutic strategy for the treatment of glioma.

\section{Materials and Methods}

2.1. Materials. The materials used are as follows: doxorubicin hydrochloride (Dalian Meilun Bio Co); NIPAM monomer $(\geq 99 \%)$ and PEI $(\mathrm{Mw}=1000 \mathrm{~g} / \mathrm{mol})$ (Aldrich Co); cell penetrating peptide (99\%, Jill Biochemical Co); glioma cells (ATCC cell bank); Dulbecco's Modified Eagle's Medium (DMEM) and fetal bovine serum (FBS) (Gibco Co, USA); cell counting kit-8 (Dojindo, Japan); UV-VIS spectrophotometer (Cary300, Varian, CA, USA); dynamic light scattering (DLS, ALV/CGS3, Germany); transmission electron microscope (Hitachi, H7000 Electron Microscope); and confocal laser scanning microscope (CLSM, Zeiss LSM 710; Carl Zeiss Meditec AG, Jena, Germany).

\subsection{Synthesis Process of DPPC}

2.2.1. Synthesis of Nanogels. PNIPAM was grafted onto the main chain of the PEI chain, and the PNIPAM-G-PEI (PPP) graft copolymer was synthesized. NIPAM monomer $(0.5 \mathrm{~g})$ was mixed with PEI $(1.0 \mathrm{~g})$ and MBA (0.5\% weight percentage of total monomer mass) in $25 \mathrm{~mL}$ water. Remove oxygen with $\mathrm{N}_{2}$, and heat to $70^{\circ} \mathrm{C}$. Tert-butyl hydroperoxides (250 L $0.01 \mathrm{M})$ were injected. The copolymer was synthesized by free radical graft copolymerization at $80^{\circ} \mathrm{C}$ under $\mathrm{N}_{2}$ protection. In general, the white solution is purified by centrifugation three times. The nanogel was redissolved in water, and the solution concentration was calculated by freeze-drying. Finally, the mother solution with a concentration of $5 \mathrm{mg}$ / $\mathrm{mL}$ was obtained and stored in the refrigerator for later use.

\subsubsection{Preparation of Nanogel Modified by Cell Penetration} Peptide (CPP). PNINAM-G-PEI $500 \mu \mathrm{L}$ was taken, and $20 \mu \mathrm{L} / \mathrm{mL}$ peptide and $200 \mu \mathrm{L}$ were added into the pump at the speed of $0.5 \mathrm{~mL} / \mathrm{h}$. After reacting for $1 \mathrm{~h}$, the solution was dialyzed with a dialysis bag (MW cutoff 1000) against PBS for $12 \mathrm{~h}$ in order to obtain DPPC solution and remove the unreacted peptide.

2.2.3. Preparation of Nanogel (DPPC) Drug Loading. The final nanogel system was configured according to the mass concentration $1: 1.1 \mathrm{mg} / \mathrm{mL}$ of DOX and $1 \mathrm{mg} / \mathrm{mL}$ of the above gel were taken, and the turbulence was shaken for 1 hour. The synthesis path is shown in Figure 1.

2.3. Morphology Characterization. Nanogels were diluted with Milli-Q water into $0.1 \mathrm{mg} / \mathrm{mL}$; then, the hydrodynamic diameter and particle size distribution of nanogel and the nanogels with NIR-light solution were evaluated by DLS at the scattering angle of $90^{\circ}$. The mother solution of the nanogel was diluted to $10 \mathrm{~g} / \mathrm{mL}$ of water and dropped onto the 


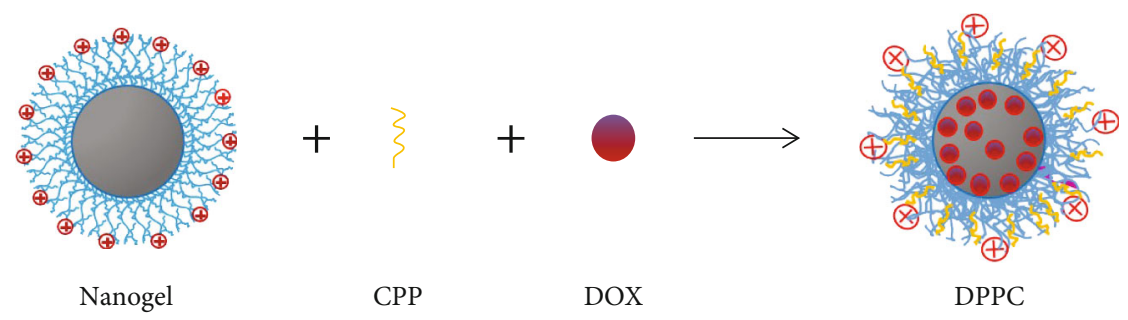

FIGURE 1: The schematic illustrating the self-assembly process of dual-targeted DPPC.

silicon chip. Its morphology was tested by scanning electron microscopy (SEM). The zeta potential was analyzed with a Zetasizer Nano ZSP by diluting the gel solution in $d i-\mathrm{H}_{2} \mathrm{O}$.

2.4. Temperature Sensitivity of PEI-PNAPAM Gel. By testing the gel at room temperature with a particle size of about $600 \mathrm{~nm}$, the particle size makes it difficult to enter the cell and harder to penetrate the $\mathrm{BBB}$. In order to verify that the carrier could achieve cross-BBB drug delivery, we further explored the particle size distribution of the gel at different temperatures. The temperature sensitivity properties of DPPC gels were measured by DLS with temperature control. The radiuses of DPPC solution (concentration $\sim 2 \mathrm{mg} / \mathrm{mL}$ ) at different temperatures were detected at the scattering angle of $90^{\circ}$.

2.5. DOX Encapsulation Efficiency. Encapsulation efficiency (EE) and the drug loading capacity (DLC) of DOX in the DPPC gels were calculated by a UV-VIS spectrophotometer at a wavelength of $485 \mathrm{~nm}$. The DPPC gels were stirred for $72 \mathrm{~h}$ at room temperature to release DOX and detected at $485 \mathrm{~nm}$ by the UV-VIS spectrophotometer. The encapsulation efficiency and the drug loading capacity of DOX in the DPPC gels were calculated according to the following equations:

$$
\begin{aligned}
& \mathrm{EE} \%=\frac{M_{E}}{M_{F}} \times 100 \%, \\
& \mathrm{LC} \%=\frac{M_{E}}{M_{T}} \times 100 \% .
\end{aligned}
$$

In the formula, $M_{E}$ means the amount of DOX wrapped in the DPPC gels, $M_{F}$ means the amount of DOX invested in the synthetic gels, and $M_{T}$ is the total weight of the DPPC gels [42, 45-48].

2.6. Serum Stability of DPPC. BSA was used as a protein in the simulated systemic circulation to detect serum stability of DPPC. The mixture solutions of BSA $(50 \mathrm{mg} / \mathrm{mL})$ and DPPC gels $(1 \mathrm{mg} / \mathrm{mL})$ in PBS were measured by DLS at the scattering angle of $90^{\circ}$.

2.7. In Vitro Drug Release. Add $4 \mathrm{~mL}$ of DPPC/DOX gel solution to the dialysis bag, and the initial DOX concentration in the gels is about $0.15 \mathrm{mg} / \mathrm{mL}$. Put the dialysis bag into a beaker, and dialyze with $1000 \mathrm{~mL}$ PBS at different temperatures. At the times $0,2,4,6,8,10,12,24,48$, and 72 hours, remove $200 \mu \mathrm{L}$ of gel solution from the dialysis bag; then, detect the accumulated DOX concentration by a UV-VIS spectrophotometer at a wavelength of $485 \mathrm{~nm}$.

$$
\mathrm{DOX}_{\text {release }}=\frac{M_{T}-M_{R}}{M_{T}} \times 100 \% \text {, }
$$

where $M_{R}$ was the DOX concentration we detected and $M_{T}$ was the initial DOX concentration of DPPC gels.

2.8. Cytotoxicity Test of DPPC at Different Temperatures. The trypsin of U87 cells was digested and counted. The cells were placed in a 96-well plate and cultured in a cell incubator at $37^{\circ} \mathrm{C}$ with $5 \% \mathrm{CO}_{2}$. By using an ultraviolet spectrophotometer instrument to detect the parcel drug synthesis system, with complete medium dilution system making the concentration of Adriamycin to $0.3 \mu \mathrm{g} / \mathrm{mL}$, cells grow to $70 \%$, sucked out of the original culture medium, DPPC/DOX system for medium, respectively, at $37^{\circ} \mathrm{C}, 39^{\circ} \mathrm{C}, 40^{\circ} \mathrm{C}$, and $42^{\circ} \mathrm{C}$ under cultivation; in each temperature setting after three holes, cultivation is done in the incubator for 24 hours. Differences in cell morphology and number were observed under the microscope among each group. The original medium was sucked out and gently washed with PBS for 2 times. 10\% CCK-8 reagent was prepared with incomplete medium, $100 \mu \mathrm{L}$ reagent was added to each group, and a blank control group was set. After the operation, 96-well plates were put into incubators (bubbles were avoided in this process as far as possible), and the reaction lasted for 1-4 hours (according to the actual situation). When the color of the medium changed to orange, we used the machine to detect.

2.9. Endocytosis Detected by LSCM. For the U87 cells in the logarithmic growth stage, $1 \times 10^{5}$ cells/well were inoculated in a confocal plate, U87 cells were seeded into confocal culture dish $\left(5 \times 10^{3}\right.$ cells/dish $)$ with $2.0 \mathrm{~mL}$ DMEM culture medium and cultured for 24 hours; then, they were treated with DPPC/DOX for $24 \mathrm{~h}$ at $37^{\circ} \mathrm{C}, 39^{\circ} \mathrm{C}, 40^{\circ} \mathrm{C}$, and $42^{\circ} \mathrm{C}$. The medium of U87 cells was piped out and rinsed with PBS twice. Then, it was fixed for $10 \mathrm{~min}$ with $1.0 \mathrm{~mL}$ paraformaldehyde solution and rinsed with PBS three times and the nucleus of U87 cells was stained with DAPI working solution for $10 \mathrm{~min}$. Finally, the U87 cells were visualized by LSCM.

2.10. In Vitro BBB Model Establishment. In vitro blood-brain barrier was established by coculture of microvascular endothelial cells and astrocytes. An appropriate amount of brain microvascular endothelial cells were taken and inoculated 


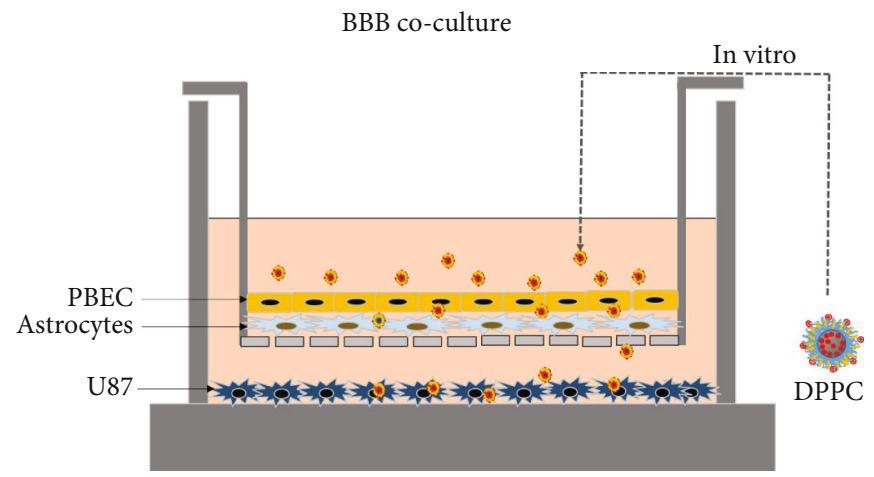

FIgURE 2: The schematic illustration of the in vitro BBB model for DPPC penetration evaluation.

on the Transwell ventricular membrane with a cell density of $1.6 \times 10^{5} / \mathrm{mL}$. An additional amount of astrocytes were taken and inoculated at the bottom of the Transwell plate with a cell density of $2.5 \times 10^{5} / \mathrm{mL} .1 .5$ and $2.5 \mathrm{~mL}$ special medium and common medium were added to the upper and lower chambers, respectively. After proliferation into monolayer and complete fusion in vitro, the cells were closely connected to each other. The astrocytes in the lower layer were used to evaluate the penetrability of substances to the PBEC layer. The establishment of the culture system is shown in Figure 2. A characteristic of the in vitro BBB model in Transwell polycarbonate membrane surface formed a uniform layer of the brain capillary endothelial cell monolayer, through the surface of astrocyte processes under film hole want to contact with brain capillary endothelial cells, are similar to simulate the BBB structure in the body, on the cell model can cultivate pools can be divided into two parts, for the pool and the pool for pool represent brain capillary lumens, the pool on behalf of the blood-brain barrier outside the brain.

2.11. BBB Penetration Ability Evaluation. DPPC/DOX with a concentration of $1 \mathrm{~mL}$ of $10 \mathrm{mg} / \mathrm{L}$ was fully dispersed and added to the Transwell upper chamber to calculate the penetration coefficient of nanogel-DOX according to the method of sodium fluorescein. The penetration coefficient of DPPC/DOX at $30 \mathrm{~min}$ was $(2.25 \pm 0.03) \times 10^{-3} \mathrm{~cm} / \mathrm{min}$, showing higher penetration than FLU at the same time point.

In addition, we seeded U87 cells in the lower compartment of the model and measured the uptake of DOX and nanogel-DOX by U87 cells in the lower compartment through flow cytometry analysis.

2.12. Statistical Analysis. The results were statistically analyzed using SPSS software, version 21.0. Unpaired $t$-test was used for statistical analysis for the comparison of the mean between the two groups, one-way ANOVA was used for statistical test for the comparison of the mean between the three groups and/or more than three groups, and the mean between the two groups was paired by LSD- $t$-test. The experimental results were expressed as the standard deviation of mean score, and $p<0.05$ was used as the criterion for the statistical difference between the groups.

\section{Results}

3.1. Preparation and Characterization of the DPPC System. As mentioned above, the physiological structure of the bloodbrain barrier (BBB) is utilized to construct a nanogel system a passive and active dual targeting as well as stable structure. In the experiments, passive targeting function as realized by thermosensitive polymer PNIPAM was conjugated to the side chain of cationic polymer PEI as reported in previous studies [49]. The grafting copolymer PEI-PNIPAM at the reaction temperature is larger than that of LCST. The hydrophobic PNIPAM will aggregate each other and form a nano structure, which will be further cross-linked by the cross-linkers, and form PNIPAM-PEI nanogels. Then, the CPP is conjugated onto the surface of this nanogel to form an active and passive targeting dual functional nanogel with positive-charged surface. Then, the chemotherapeutic drug doxorubicin (DOX) will be loaded into the nanogel by physical mixing resulting in an intelligent delivery system DOX@PNIPAM-PEI-CPP (DPPC) as schemed in Figure 1. More importantly, the BBB penetrability mechanism of DPPC will be detailedly investigated and illustrated by using an in vitro BBB model. The drug delivery system designed in this study provides a new therapeutic strategy for the treatment of glioma.

The size and size distribution of the DPPC were then evaluated by DLS as shown in Figure 3(a). It can be seen from Figure 3(a) that the hydrodynamic radius of DPPC is around $300 \mathrm{~nm}$. This size is coincided to that obtained in TEM as shown in Figure 3(b). Both the TEM and DLS further confirm its well spherical morphology and uniform distribution, which is attributed to the cross-linked 3D structure as finely regulated in the radical polymerization process. Such stable structure is essential for promoting in vivo circulation.

On the other hand, the cytotoxicity of the nanogel should be as low as possible because the PEI is known to be toxic to cells by the membrane breaking. However, after the PNIAPM modification, the cytotoxicity of PEI decreases obviously due to the low potential. The cytotoxicity against nontumor cells (HEK293T) is shown in Figure 3(c), which indicates that the DPPC is a biocompatible carrier for further application. In addition, it is known that a weak cationic surface is preferable for enhancing the $\mathrm{BBB}$ penetration by the cell membrane merging. Here, the surface properties especially the surface potential of DPPC are tested by the Zetasizer Nano ZSP as 


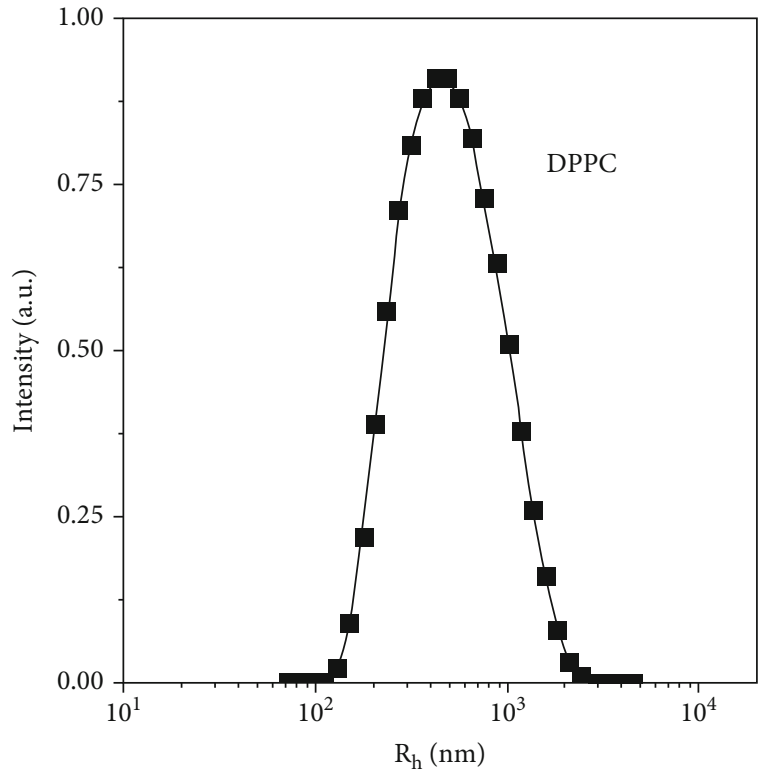

(a)

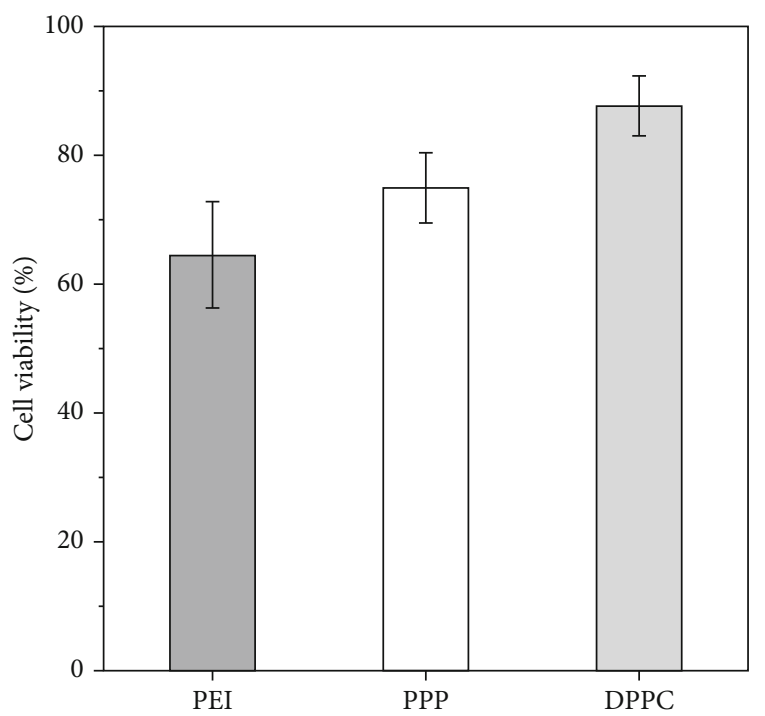

(c)

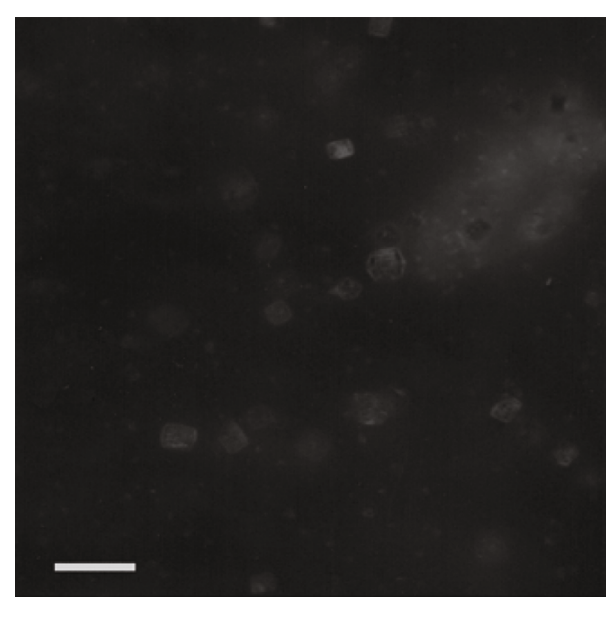

(b)

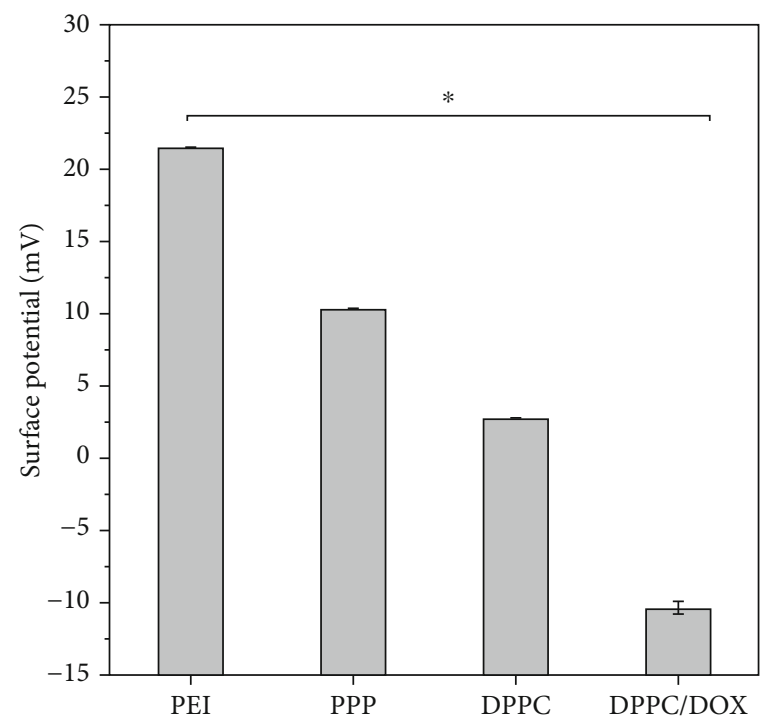

(d)

FIGURe 3: The size and size distribution of DPPC (a); TEM image of DPPC/DOX (b); the cytotoxicity of PEI, PPP, and DPPC (c); the surface potential of different groups (d).

shown in Figure 3(d). The potential of PEI is about $30 \mathrm{mV}$, which decreases to about $0-5 \mathrm{mV}$ step to step. All these indicate that this nanogel is an excellent candidate for the BBB penetration as it arrives at the tumor.

3.2. The Passive and Active Dual-Targeting Evaluation. It is known that the LCST of PNIPAM is about $32^{\circ} \mathrm{C}$, which can be further increased or decreased by the hydrophilic or hydrophobic modification for different applications. In this study, the composition and structure of PNIPAM-PEI nanogel are the same as the previous study [47]. Although it was hydrophilically modified by PEI, the LCST of the nanogel is still about $32^{\circ} \mathrm{C}$ because the PNIPAM chains are introduced to PEI and aggregation together to form a PNIPAM core. The PNIPAM chain backbone has little changes as a result of similar LCST as PNIAPM as shown in Figure 4(a). Noted here, the passive targeting will be realized by the thermosensitive PNIPAM polymer as the temperature larger than that of LCST through the TSPT as illustrated above [41, 42].

When the tumor temperature is higher than the volume phase change temperature (VPTT), the thermosensitive polymer- (PNIPAM) based nanogel can enhance the absorption of cells and increase the aggregation in the tumor, and this passive targeting significantly promotes tumor inhibition. The in vitro cytotoxicity of DPPC is shown in Figure 4(b). The cytotoxicity increases as the temperature clearly indicates the TSPT function, which can be utilized to enhance the BBB penetration as described in the following. As mentioned above, the CPP peptide is utilized to break the cellular membrane. In this study, such CPP is mainly for the astrocyte which is 


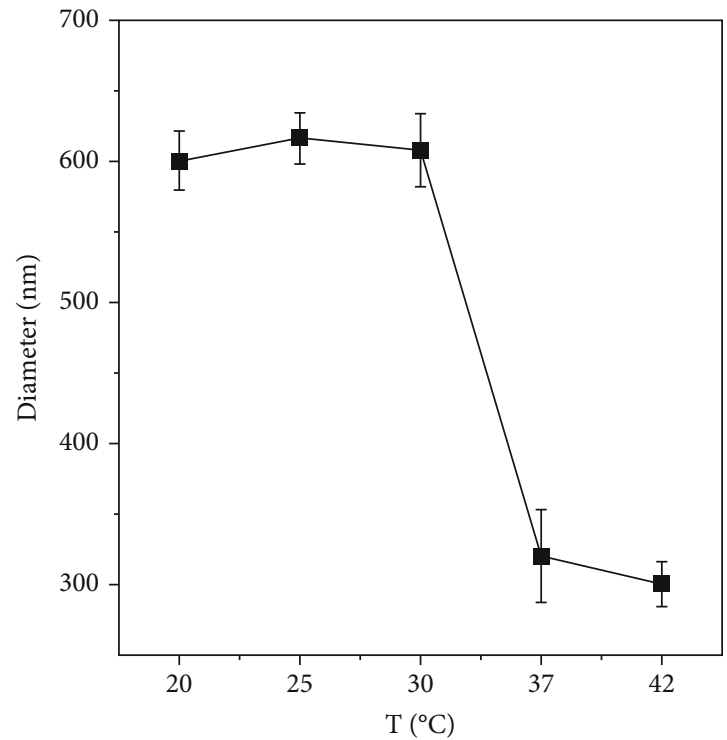

(a)

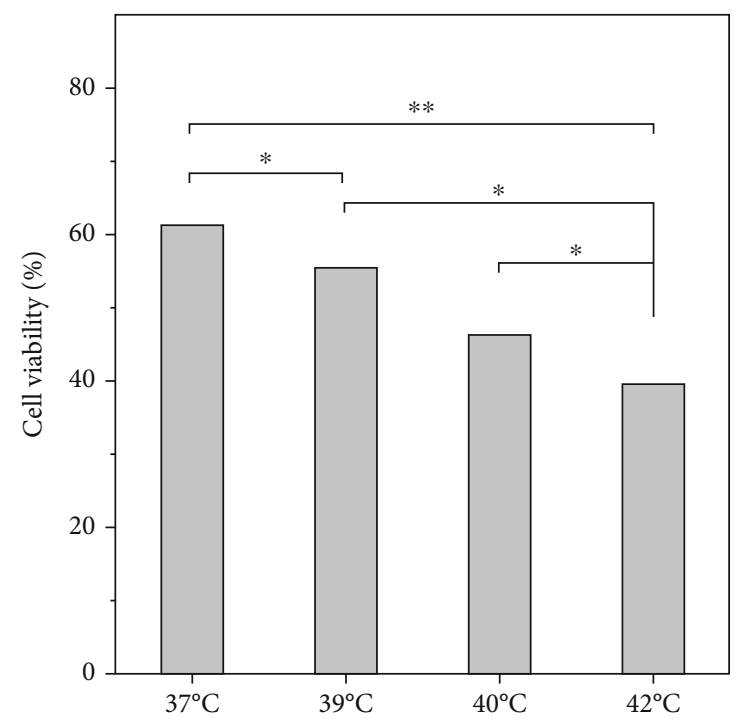

(b)

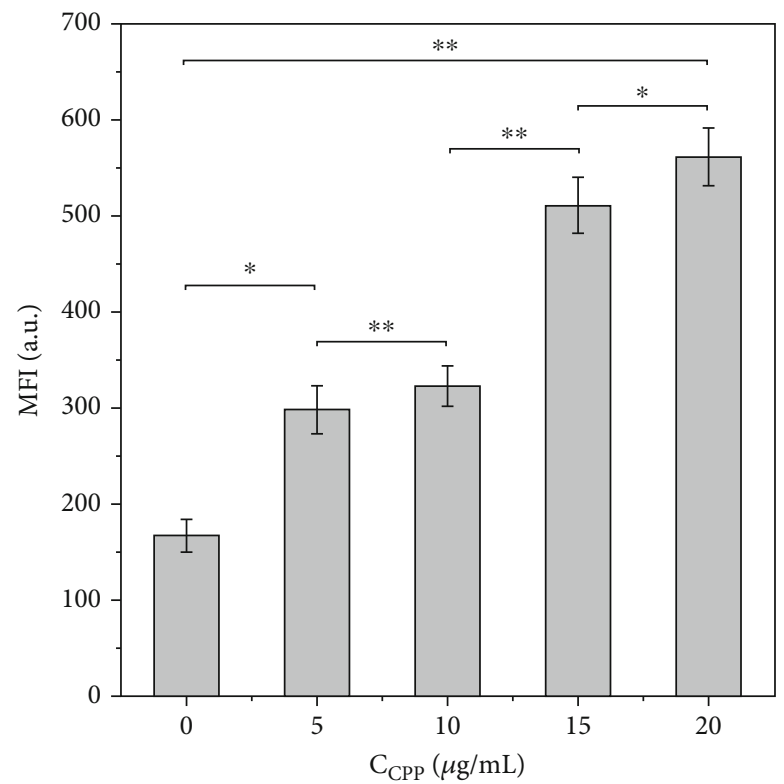

(c)

Figure 4: The temperature dependence of the size of DPPF (a); the temperature dependence of the cytotoxicity of DPPC (b); the fluorescent intensity of DOX at different CPP concentrations (c).

the main part of the $\mathrm{BBB}$. If the $\mathrm{CPP}$ can assist the nanogel to penetrate through the astrocyte, the CPP will increase the BBB penetration ability. A fluorescent microscopy is conducted to evaluate this BBB penetration ability of DPPC with CPP onside the surface. The cellular level interaction between DPPC and astrocyte cells is shown in Figure 4(c).

3.3. The In Vitro Serum Stability and Drug Release Profile. As the DPPC accumulated in tumor cells, the successful DOX release is important for the glioblastoma therapy. In this study, the thermosensitive PNIPAM-based nanogel will not only promote the U87 tumor cells passive targeting but also enhance the drug release inside tumor due to the well-known high temperature of tumor microenvironment as reported previously
$[32,41,42]$. Thus, the temperature-enhanced drug release profile is evaluated as shown in Figure 5(b). The volume phase transition temperature (VPTT) of DPPC is about $32^{\circ} \mathrm{C}$ as shown in Figure 4(a). Noted here, the transition temperature of DPPC is used as VPTT but not LCST because the temperature is tested by UV and monitored by the absorption of the DPPC solution. So the temperature is the transmission of DPPC which reflects the inter-DPPC aggregation after the phase transition of PNIPAM as T > VPTT [32, 41, 42]. When $T>$ VPTT, the drug release increases sharply, which is attributed to the shrinking of DPPC as a result of increased osmotic pressure and high drug diffusion from DPPC. This high drug release is also indirectly reflected in the enhanced cytotoxicity of DPPC against tumor cell as shown in Figure 4. 


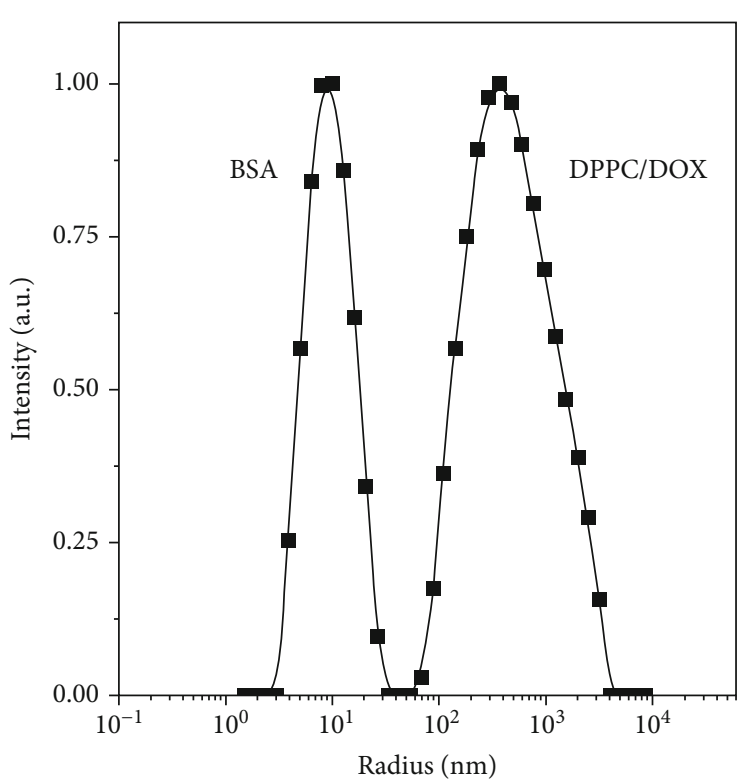

(a)

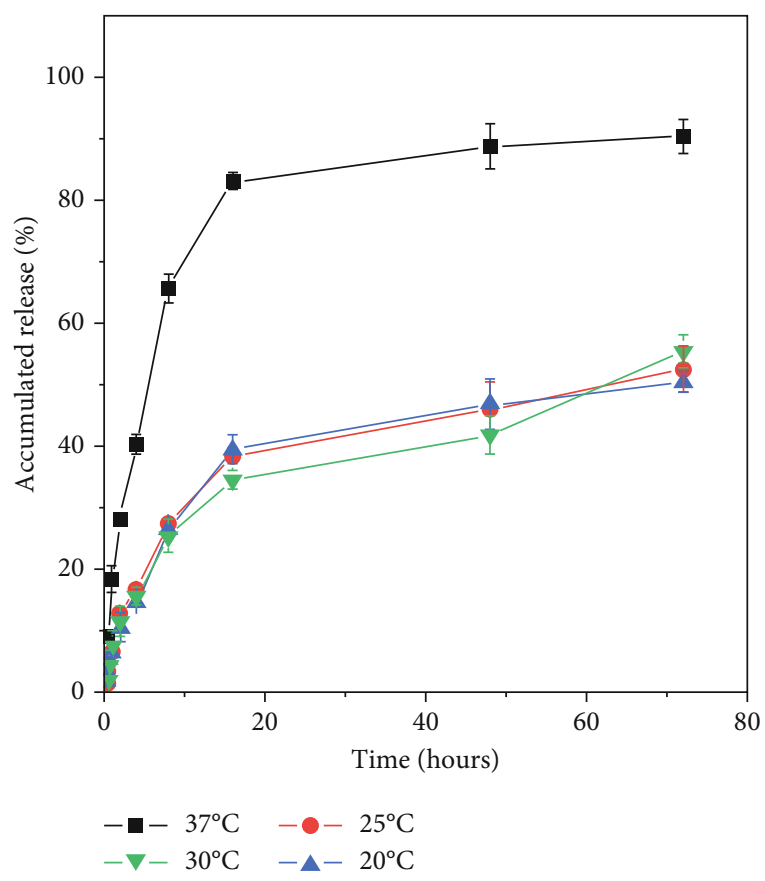

(b)

FIGURE 5: The serum stability of DPPC as evaluated by DLS (a). The effects of temperature on the drug release profile (b).

On the other hand, the serum stability plays an important role in the in vivo drug delivery. As reported in previous studies, the serum stability of nanomedicine can be evaluated by the DLS. In the experiment, DPPC nanogel is mixed with the serum proteins. Then, the DLS is used to detect the size and size distribution of BSA, DPPC/DOX, and their blends in solutions. It is well-known that the size of BSA is about $10 \mathrm{~nm}$ (data not shown here). Figure 5(a) shows the size and size distribution of the BSA and DPPC blends. It is found that the size of BSA is well separated from that of the DPPC. The two distinct peaks indicate the little interaction between the BSA and DPPC which includes absorption of BSA onto the DPPC.

3.4. Successful In Vitro BBB Penetration by DPPC. For further evaluation the BBB penetration ability of DPPC as constructed in this study, the in vitro BBB model is set up as shown in Figure 2. Here, the upper-cubicle is used to culture the astrocyte cells, which mainly compose astrocyte for outside barrier mimicking for BBB. In the experiments, the DPPC is cultured with the astrocyte in this up cell for evaluating its penetration ability. Once DPPC successfully penetrates the astrocyte-based barrier, it will go down to the lower cell, where the gliomas (U87) are cultured in medium. Given the DPPC pass through the BBB and cocultured with U87 cells, it will be internalized by tumor cell following by the DOX release. Then, the U87 will be killed by the drug with low viability in the CCK- 8 assay.

The effects of temperature and CPP on the astrocyte-based barrier penetration are investigated by the FCM. Firstly, the temperature dependence of $\mathrm{BBB}$ penetration ability is evaluated by tuning the temperature of the setup in a water bath. The fluorescence intensity in the U87 cells is shown in Figure 6(a). It is easy to find that the penetration ability of
DPPC increases as the temperature increases. As mentioned above, this increased MFI is attributed to two factors. One is the many factors which come from the passive targeting of DPPC to the tumor cells named as TSPT. Another is partly due to the temperature-enhanced drug release. On the other hand, the CPP also plays an important role in the BBB penetration as mentioned above. The effects of CPP on the astrocyte-based barrier penetration are also evaluated by monitoring the DOX concentration through flow cytometry analysis as shown in Figure 6(b). The DOX content in the control group has a $C$ of about $2 \mathrm{mg} / \mathrm{mL}$; the DOX obtained from DPPC group is about $32.5 \mathrm{mg} / \mathrm{mL}$, as compared with the drug the concentration obtained in the tumor cells which is much higher than that of DPPC. This increased drug concentration is also attributed to two factors. One is the DPPC enhances the cell uptake. Another is attributed to the CPP, which is the main factor by successfully breaking the cell membrane as mentioned above.

Subsequently, the cytotoxicity of DPPC against U87 cells is then tested by the CCK- 8 assay for further confirming the BBB penetration ability. The effects of temperature-regulated TSPT (passive targeting) and CPP-mediated active targeting on the cell viability are shown in Figure 6(c). It is clearly shown that, as compared with the control, the viability of U87 cells decreases as the CPP is introduced onto the nanogel surface (DPPC of the second column). In addition, this cell viability of DPPC at $T>$ VPTT is even lower than that of DPPC at room temperature. Taken together, it is helpful to conclude that the astrometry-based barrier penetration ability can be increased by introducing thermosensitive PNIPAM polymer to the nanogel for enhancing its TSPT. On the other hand, this $\mathrm{BBB}$ penetration ability of nanogel can be further enhanced by linking the CPP onto the surface. 


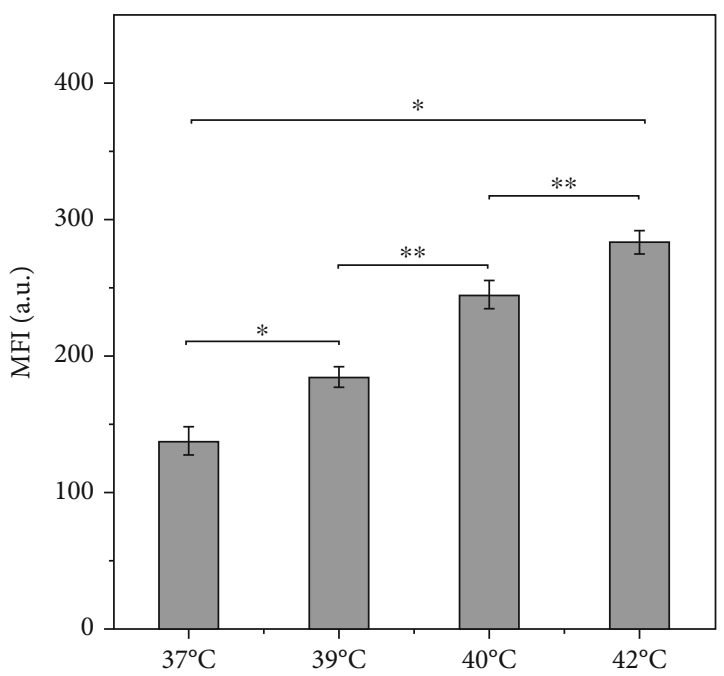

(a)

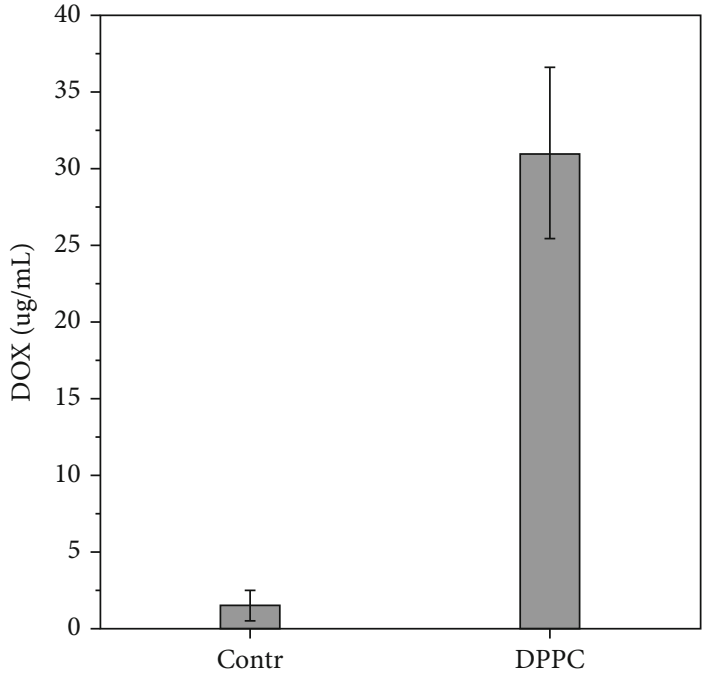

(b)

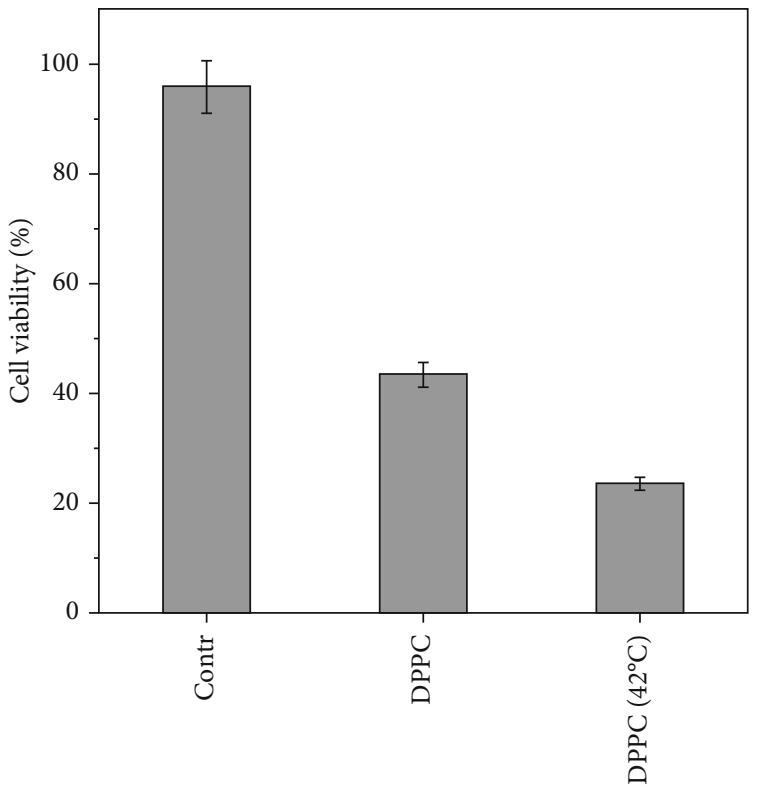

(c)

FIgURE 6: The temperature dependence of BBB penetration ability of DPPC (a); the effect of CPP on the BBB penetration ability (b); the tumor cell (U87) viability cultured in the lower chamber in the BBB model as evaluated by the CCK- 8 assay (c).

\section{Discussion}

Glioblastoma is the most fatal brain tumor in adults. Recent developments in molecular medicine have improved its original pathology. In success through BBB treatment, the clinical application faces the biggest challenges including poor drug targeting because of the low blood-brain barrier (BBB) permeability and/or rapid elimination resulting in most of the central nervous system drugs, $1-4 \%$. Clinical alternative methods like the in-site injection of drugs may be caused by physical BBB damage which will enhance toxins to the brain including the neuronal dysfunction or incidence of central nervous system infection. Therefore, the ideal method of blood-brain barrier penetration should be controllable, transient, reversible, spe- cific, and low toxic. Although many strategies associated with nanosized delivery system shave been developed for overcoming the BBB and glioblastoma treatment [24-40], new nanosized particles with better application potential still need to be developed. In this paper, we developed a new nanosized drug delivery system for glioblastoma treatment, and the DPPC drug delivery system designed in this study can effectively overcome the shortcomings that appeared in traditional treatments with high BBB penetration and low side effects. Our DPPC drug delivery system was designed based on our previous study on transporting protein drug system with thermosensitive passive targeting function (TSPT) [42-44], and the combination of thermosensitive PNIPAM and CPP in chemical drug delivery for $\mathrm{BBB}$ overcoming and glioblastoma treatment also has 


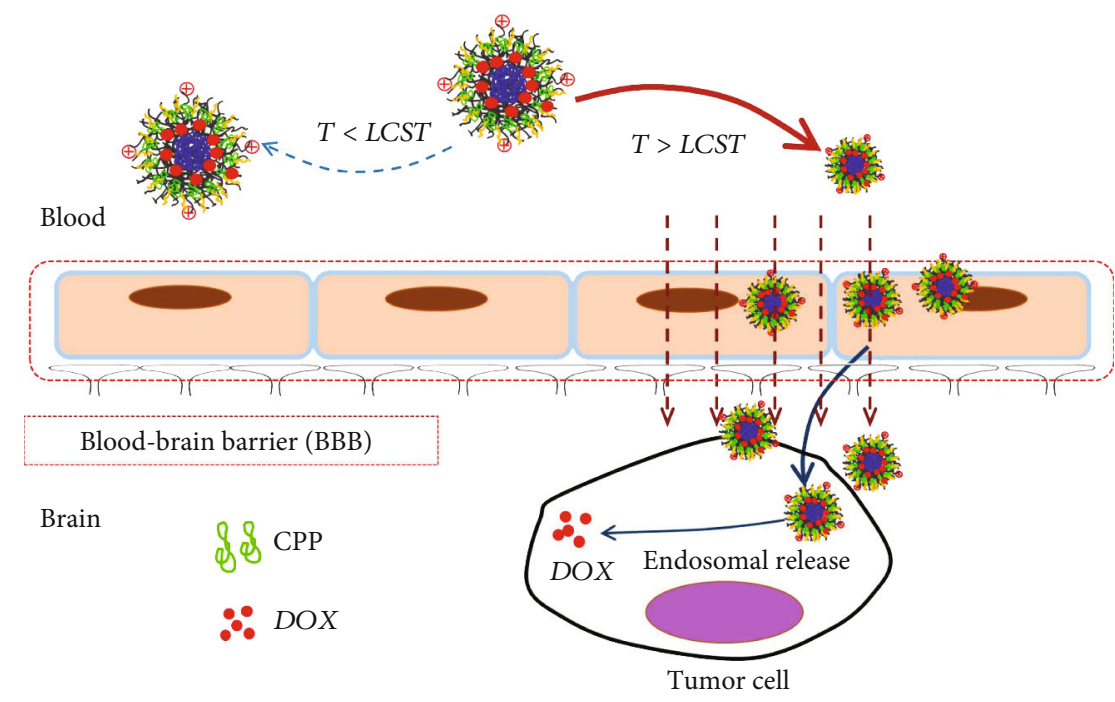

FIGURE 7: The scheme illustrating the detailed mechanism about in vitro BBB penetration ability of DPPC.

innovation at some degree. As illustrated above, the DPPC is constructed by introducing thermosensitive PNIPAM to the side chain of PEI which can assemble for a core shell structural nanogel. Then, the CPP is linked onto the surface of the nanogel resulting in temperature and biotargeting dual functions. The 3D cross-linking structure with mass CPP surrounding the surface offers DPPC high serum stability and compatibility.

As we know, the PNIPAM inside core of DPPC shows the temperature-sensitive passive targeting (TSPT). On the other hand, the CPP onside of the nanogel results in an active targeting function. The chemotherapeutic drug doxorubicin (DOX) will be loaded into the nanogel resulting in an intelligent delivery system DOX@PNIPAM-PEI-CPP (DPPC). The introduction of biocompatible PINPAM to the PEI branch chain reduces the surface potential and thus reduces the toxicity. More importantly, the BBB penetrability mechanism of DPPC is thus obviously enhanced by this passive and active dualtargeting functional nanogel. The detailed penetrating mechanism is schemed in Figure 7. The obviously enhanced BBB penetration ability is mainly attributed to two factors. One is the astrocyte cellular membrane breaking by the active targeting $\mathrm{CPP}$, which strongly promotes the BBB across ability with large intratumor accumulation of DPPC. On the other hand, it is well-known that the normal organism's temperature is about $37^{\circ} \mathrm{C}$, while the temperature of the tumor microenvironment is around $42^{\circ} \mathrm{C}$, which is larger than that of VPTT or DPPC. So when the DPPC arrives at the tumor cell, the TSPT promotes the internalization of DPPC by U87 cells. The enhanced tumor cell's uptake further promotes the intracellular drug concentration which induces the high tumor cellkilling efficiency. All these well coincide with the results as investigated through the in vitro $\mathrm{BBB}$ model.

\section{Conclusion}

In this study, we designed a thermally sensitive and CPP conjugating dual-targeted nanogel delivery system DPPC. The in vitro-constructed BBB-model showed that DPPC can efficiently penetrate the $\mathrm{BBB}$ and concentrate in the tumor site through the TSPT and astrocyte cellular membrane breaking. It was found that doxorubicin (DOX) was successfully in-site released out by such functional DPPC automatically attributed to the relative high temperature inside tumor microenvironment (TME) $\left(\sim 42^{\circ} \mathrm{C}\right)$, which obviously improved intratumor drug accumulation and tumor cell-killing effects. The drug delivery system designed in this study provides a new therapeutic strategy for the treatment of glioma.

\section{Data Availability}

All data is presented in the article, with no other data sources or web links.

\section{Conflicts of Interest}

The authors declare that they have no conflicts of interest.

\section{Authors' Contributions}

Jun Liu, Meng Li, Yong Huang, and Li Zhang are co-first authors.

\section{Acknowledgments}

This work was financially supported by the National Natural Science Foundation of China (81802714, 92059112); the National Key New Drug Creation and Manufacturing Program, Ministry of Science and Technology (2018ZX09J181074); and the Youth Start-up Fund of Naval Medical University (2018QN0003). And Ms. Yingying Zhang of Changhai Hospital should be appreciated for her kind efforts.

\section{References}

[1] D. Sturm, S. Bender, D. T. W. Jones et al., "Paediatric and adult glioblastoma: multiform (epi)genomic culprits emerge," Nature Reviews Cancer, vol. 14, no. 2, pp. 92-107, 2014.

[2] Y. Liu, H. Liu, H. Yan et al., “Aggregation-induced absorption enhancement for deep near-infrared II photoacoustic imaging 
of brain gliomas in vivo," Advanced Science, vol. 6, no. 8, p. 1801615, 2019.

[3] N. Cohen and R. O. Weller, "WHO Classification of Tumours of the Central Nervous System (4th edition)," Neuropathology and Applied Neurobiology, vol. 33, no. 6, pp. 710-711, 2007.

[4] H. Ohgaki and P. Kleihues, "Genetic pathways to primary and secondary glioblastoma," The American Journal of Pathology, vol. 170, no. 5, pp. 1445-1453, 2007.

[5] Q. T. Ostrom, H. Gittleman, P. Liao et al., "CBTRUS statistical report: primary brain and central nervous system tumors diagnosed in the United States in 2007-2011," Neuro-oncology, vol. 16, supplement 4, pp. iv1-iv63, 2014.

[6] R. Stupp, W. P. Mason, M. J. van den Bent et al., "Radiotherapy plus concomitant and adjuvant temozolomide for glioblastoma," New England journal of medicine, vol. 352, no. 10, pp. 987-996, 2005.

[7] R. Stupp, M. E. Hegi, W. P. Mason et al., "Effects of radiotherapy with concomitant and adjuvant temozolomide versus radiotherapy alone on survival in glioblastoma in a randomised phase III study: 5-year analysis of the EORTC-NCIC trial," The lancet oncology, vol. 10, no. 5, pp. 459-466, 2009.

[8] M. L. Guion-Almeida and A. Richieri-Costa, "CNS midline anomalies in the opitz G/BBB syndrome: report on 12 Brazilian patients," American Journal of Medical Genetics, vol. 43, no. 6, pp. 918-928, 1992.

[9] H. Peluffo, U. Unzueta, M. L. Negro-Demontel et al., "BBBtargeting, protein-based nanomedicines for drug and nucleic acid delivery to the CNS," Biotechnology Advances, vol. 33, no. 2, pp. 277-287, 2015.

[10] K. Agata, Y. Soejima, K. Kato, C. Kobayashi, Y. Umesono, and K. Watanabe, "Structure of the planarian central nervous system (CNS) revealed by neuronal cell markers," Zoological science, vol. 15, no. 3, pp. 433-440, 1998.

[11] H. N. Zhen, X. Zhang, P. Z. Hu et al., "Survivin expression and its relation with proliferation, apoptosis, and angiogenesis in brain gliomas," Cancer, vol. 104, no. 12, pp. 27752783, 2005.

[12] S. Pacioni, Q. G. D'Alessandris, M. Buccarelli et al., "Brain invasion along perivascular spaces by glioma cells: relationship with blood-brain barrier," Cancers, vol. 12, no. 1, 2019.

[13] W. M. Pardridge, "Blood-brain barrier drug targeting: the future of brain drug development," Molecular interventions, vol. 3, no. 2, pp. 90-105, 2003.

[14] J. Huse and E. Holland, "targeting brain cancer: advances in the molecular pathology of malignant glioma and medulloblastoma," Nature reviews cancer, vol. 10, no. 5, pp. 319-331, 2010.

[15] N. J. Abbott, A. A. K. Patabendige, D. E. M. Dolman, S. R. Yusof, and D. J. Begley, "Structure and function of the bloodbrain barrier," Neurobiology of Disease, vol. 37, no. 1, pp. 1325,2010 .

[16] B. T. Hawkins and T. P. Davis, "The blood-brain barrier/neurovascular unit in health and disease," Pharmacological reviews, vol. 57, no. 2, pp. 173-185, 2005.

[17] P. Ballabh, A. Braun, and M. Nedergaard, "The blood-brain barrier: an overview: structure, regulation, and clinical implications," Neurobiology of disease, vol. 16, no. 1, pp. 1-13, 2004.

[18] R. D. Oehring, M. Miletic, M. M. Valter et al., "Vascular endothelial growth factor (VEGF) in astrocytic gliomas-a prognostic factor?," Journal of neuro-oncology, vol. 45, no. 2, pp. 117125, 1999.
[19] N. Ortuzar, E. G. Argandoña, H. Bengoetxea, O. Leis, S. Bulnes, and J. V. Lafuente, "Effects of VEGF administration or neutralization on the BBB of developing rat brain," ACTA NEUROCHIRURGICA's Supplement, vol. 106, 2010.

[20] A. G. de Boer and P. J. Gaillard, "Drug targeting to the brain," Annual Review of Pharmacology and Toxicology, vol. 47, no. 1, pp. 323-355, 2007.

[21] Y. Yan, K. Zhou, H. Xiong et al., "Aerosol delivery of stabilized polyester-siRNA nanoparticles to silence gene expression in orthotopic lung tumors," Biomaterials, vol. 118, pp. 84-93, 2017.

[22] W. Pardridge, "BBB-genomics: creating new openings for brain-drug targeting," Drug Discovery Today, vol. 6, pp. 381383, 2001.

[23] B. Prasad and J. D. Unadkat, "The concept of fraction of drug transported $\left(f_{t}\right)$ with special emphasis on BBB efflux of CNS and antiretroviral drugs," Clinical Pharmacology \& Therapeutics, vol. 97, no. 4, pp. 320-323, 2015.

[24] O. van Tellingen, B. Yetkin-Arik, M. C. de Gooijer, P. Wesseling, T. Wurdinger, and H. E. de Vries, "Overcoming the blood-brain tumor barrier for effective glioblastoma treatment," Drug Resistance Updates, vol. 19, pp. 1-12, 2015.

[25] M. Da Ros, V. De Gregorio, A. L. Iorio et al., "Glioblastoma chemoresistance: the double play by microenvironment and blood-brain barrier," International journal of molecular sciences, vol. 19, no. 10, p. 2879, 2018.

[26] L. Cucullo, B. Aumayr, E. Rapp, and D. Janigro, "Drug delivery and in vitro models of the blood-brain barrier," Current Opinion in Drug Discovery \& Development, vol. 8, 2005.

[27] K. Hynynen, N. McDannold, N. Vykhodtseva, and F. A. Jolesz, "Non-invasive opening of BBB by focused ultrasound," ACTA NEUROCHIRURGICA's Supplement, vol. 86, pp. 555-558, 2003.

[28] N. Hu, X. Shi, Q. Zhang et al., "Special interstitial route can transport nanoparticles to the brain bypassing the bloodbrain barrier," Nano Research, vol. 12, no. 11, pp. 2760-2765, 2019.

[29] W. Li, S.-S. Feng, and Y. Guo, "Block copolymer micelles for nanomedicine," Nanomedicine, vol. 7, pp. 169-172, 2012.

[30] W. Li, S. Feng, and Y. Guo, "Tailoring polymeric micelles to optimize delivery to solid tumors," Nanomedicine, vol. 7, no. 8, pp. 1235-1252, 2012.

[31] W. Li, M. Nakayama, J. Akimoto, and T. Okano, "Effect of block compositions of amphiphilic block copolymers on the physicochemical properties of polymeric micelles," Polymer, vol. 52, no. 17, pp. 3783-3790, 2011.

[32] D. Chen, X. Zhu, W. Tao et al., "Regulation of pancreatic cancer microenvironment by an intelligent gemcitabine@nanogel system via in vitro 3D model for promoting therapeutic efficiency," Journal of Controlled Release, vol. 324, pp. 545-559, 2020.

[33] J. Mo, L. He, B. Ma, and T. Chen, “Tailoring particle size of mesoporous silica nanosystem to antagonize glioblastoma and overcome blood-brain barrier," ACS applied materials \& interfaces, vol. 8, no. 11, pp. 6811-6825, 2016.

[34] K. Ulbrich, T. Hekmatara, E. Herbert, and J. Kreuter, "Transferrin- and transferrin-receptor-antibody-modified nanoparticles enable drug delivery across the blood-brain barrier (BBB)," European Journal of Pharmaceutics and Biopharmaceutics, vol. 71, no. 2, pp. 251-256, 2009.

[35] L. Chen, J. Chen, S. Qiu et al., "Biodegradable nanoagents with short biological half-life for SPECT/PAI/MRI multimodality 
imaging and PTT therapy of tumors," Small, vol. 14, no. 4, 2018.

[36] P. Zhang, Y. Hou, J. Zeng et al., "Coordinatively unsaturated $\mathrm{Fe}^{3+}$ based activatable probes for enhanced MRI and therapy of tumors," Angewandte Chemie International Edition, vol. 58, no. 32, pp. 11088-11096, 2019.

[37] P. Zhang, J. Meng, L. Yingying, Z. Wang, and Y. Hou, "pHsensitive ratiometric fluorescent probe for evaluation of tumor treatments," Materials, vol. 12, p. 1632, 2019.

[38] C. Xu, Y. Yan, J. Tan et al., "Biodegradable nanoparticles of polyacrylic acid-stabilized amorphous $\mathrm{CaCO}_{3}$ for tunable pH-responsive drug delivery and enhanced tumor inhibition," Advanced Functional Materials, vol. 29, no. 24, p. 1808146, 2019.

[39] Z. Li, X. Wei, and T. Ngai, "Controlled production of polymer microspheres from microgel-stabilized high internal phase emulsions," Chemical Communications, vol. 47, no. 1 , pp. 331-333, 2011.

[40] Z. Zhao, L. Luan, X. Wei et al., "Nanoelectronic coating enabled versatile multifunctional neural probes," Nano Letters, vol. 17, no. 8, pp. 4588-4595, 2017.

[41] F. Zhang, Y. Hou, M. Zhu et al., "Death pathways of cancer cells modulated by surface molecule density on gold nanorods," Advanced Science, vol. 15, p. 2102666, 2021.

[42] W. Li, J. Li, J. Gao et al., "The fine-tuning of thermosensitive and degradable polymer micelles for enhancing intracellular uptake and drug release in tumors," Biomaterials, vol. 32, no. 15, pp. 3832-3844, 2011.

[43] H. Li, G. Zhang, C. Jiang et al., "Suppression of rituximabresistant B-cell lymphoma with a novel multi-component anti-CD20 mAb nanocluster," Oncotarget, vol. 6, no. 27, pp. 24192-24204, 2015.

[44] H. Li, Y. Sun, D. Chen et al., "Synergistic anti-tumor therapy by a comb-like multifunctional antibody nanoarray with exceptionally potent activity," Scientific reports, vol. 5, no. 1, p. 15712, 2015.

[45] X. Zhu, Y. Sun, D. Chen et al., "Mastocarcinoma therapy synergistically promoted by lysosome dependent apoptosis specifically evoked by 5-Fu@nanogel system with passive targeting and $\mathrm{pH}$ activatable dual function," Journal of Controlled Release, vol. 254, pp. 107-118, 2017.

[46] W. Li, H. Zhao, W. Qian et al., "Chemotherapy for gastric cancer by finely tailoring anti-Her2 anchored dual targeting immunomicelles," Biomaterials, vol. 33, no. 21, pp. 53495362, 2012.

[47] W. Li, S. S. Feng, and Y. Guo, "Polymeric nanoparticulates for cancer immunotherapy," Nanomedicine, vol. 8, no. 5, pp. 679682, 2013.

[48] L. Chen, F. Chen, M. Zhao et al., "A redox-sensitive micellelike nanoparticle self-assembled from amphiphilic adriamycin-human serum albumin conjugates for tumor targeted therapy," BioMed research international, vol. 2015, Article ID 987404, 2015.

[49] P. Cao, X. Sun, Y. Liang et al., "Gene delivery by a cationic and thermosensitive nanogel promoted established tumor growth inhibition," Nanomedicine, vol. 10, no. 10, pp. 1585-1597, 2015. 